\title{
Revisión de herramientas de asistencia en la selección de soluciones constructivas sostenibles de edificación
}

\author{
Review of tools to assist in the selection of sustainable \\ building assemblies
}

P. Huedo(*), B. López-Mesa ${ }^{(* *)}$

\section{RESUMEN}

Este artículo presenta un estado del arte de las herramientas existentes a nivel internacional de asistencia en la selección de soluciones constructivas sostenibles de edificación basadas en el Análisis de Ciclo de Vida (ACV), identificándose las características de estas que pueden ayudar a los proyectistas en las fases iniciales de diseño. En una primera fase, se clasifican las herramientas existentes. En una segunda fase, analizamos en detalle las herramientas mejor valoradas por los autores del artículo como apoyo al proyectista en las fases iniciales del proyecto. En este análisis se describen las ventajas y limitaciones que presentan estas herramientas. Como conclusión, se elabora un listado de directrices para el desarrollo, en España, de una herramienta que sirva de apoyo al proyectista para la selección de materiales y soluciones constructivas en la fase de diseño.

Palabras clave: Edificación sostenible; herramientas de evaluación medioambiental de edificios; análisis de ciclo de vida; selección de materiales; selección de soluciones constructivas.

\section{SUMMARY}

This paper presents a survey of the stateof-the-art in tools to assist in the selection of sustainable building assemblies based on Life Cycle Analysis (LCA). The tools are analysed to identify the characteristics that make them suitable to assist in the early phases of design. In a first phase of analysis, the existing tools are classified. In a second phase of analysis, the tools found to be more suitable to assist in building design are further analysed, by describing their advantages and limitations. In conclusion, a list of guidelines for the development of a Spanish tool to assist building designers in the selection of sustainable building assemblies is proposed.

Keywords: Sustainable building; building environmental assessment tools; life cycle analysis; materials selection; building assemblies selection.

\footnotetext{
(*) Universitat Jaume I. Castellón (España).

(**) Universidad de Zaragoza (España).

Persona de contacto/Corresponding author: belinda@unizar.es (B. López-Mesa).
}

Recibido/Received: 28 jul 2011 Aceptado/Accepted: 04 nov 2011 Publicado online/ Published online: 28 jan 2013 


\section{INTRODUCCIÓN}

A partir de 1987, fecha en que se publicó el informe Brundtland elaborado para la ONU y se utilizó por primera vez el término "desarrollo sostenible", definido como "el desarrollo que satisface las necesidades del presente sin comprometer las necesidades de las futuras generaciones" (1), la protección ambiental ha pasado a convertirse en una necesidad global y crece la urgencia por incorporar a todos nuestros procesos productivos los principios de sostenibilidad.

La construcción es responsable de la utilización indiscriminada de recursos no renovables y de una importante fuente de residuos y contaminación para el aire, el suelo y el agua. Según datos de la UNEP (Programa de las Naciones Unidas para el Medio Ambiente) y OCDE (Organización para la Cooperación y el Desarrollo Económicos) (2), el entorno edificado, representa un consumo de energía del 25 al $40 \%$, una carga de residuos sólidos del 30 al $40 \%$ y una carga de emisión de gases de efecto invernadero del 30 al 40\%. Es imprescindible que la construcción llegue a ser sostenible y para ello ha de estar basada no sólo en la correcta gestión de residuos y conservación de la energía, ya reconocidos en la normativa española (3) (4) (5), sino también en el uso sostenible de los recursos naturales, estrategia ya contemplada desde la Unión Europea (6). Para ello sería necesario incorporar mecanismos de cuantificación y de prevención de los impactos producidos por el empleo de diferentes soluciones constructivas teniendo en cuenta todo el ciclo de vida. Así, González (7) recuerda las palabras de Steadman Philiph Steadman en 1975: "Si se concibe el edificio como un sistema completo que utiliza energía y materiales a lo largo de su ciclo vital, el análisis debería empezar con la extracción de materias primas empleadas en la construcción, incluir su transporte y colocación, y luego, al final de la vida del edificio, cuando se derribe, debería tenerse en cuenta cómo se dispersan los materiales y se vuelven a utilizar". Uno de los aspectos claves en todo proceso constructivo es el diseño del edificio, ya que durante esta fase, se deben tomar múltiples decisiones que afectan al resultado final. En las primeras etapas del proceso constructivo los proyectistas pueden tomar decisiones para mejorar los resultados medioambientales (8). El diseño es una fase singularmente crucial para la incorporación de medidas que fomenten la sostenibilidad, ya que determina y condiciona las actividades que se desarrollarán posteriormente (9).
El diseñador o proyectista como vertebrador del eje para el posterior desarrollo o fabricación del edificio debe ser capaz de controlar adecuadamente la elección de los materiales y de las soluciones constructivas empleadas en su proyecto (10), para ello ha de considerar una serie de variables que le obligan a tomar decisiones en la fase de diseño que afectarán a la viabilidad del producto $y$ al resultado final.

Sería de gran ayuda poder identificar y evaluar los impactos ambientales antes de que se produzcan, por ejemplo, disponiendo de bases de datos de materiales y de soluciones constructivas catalogadas y valoradas según criterios de sostenibilidad reconocidos. Estas bases de datos deberían permanecer abiertas para poder ser actualizadas y ampliadas permanentemente. En España, se comenzó desarrollando bases de datos de materiales de construcción sostenibles (11). Ahora bien, la forma en que se obtiene la puntuación medioambiental en estas bases de datos no está basada en ningún método científico ampliamente aceptado, y los materiales son estudiados como elementos individuales, y no como elementos que trabajan conjuntamente para realizar una cierta función en una solución constructiva, lo que podría conducir a una decisión errónea al utilizar un material que por sí solo tiene un buen comportamiento ambiental, pero que requiere la utilización de otros materiales más perjudiciales para formar parte de una solución constructiva. Además el impacto medioambiental de una solución constructiva no sólo depende de los materiales de que se compone, sino también del proceso de construcción, de los requerimientos de mantenimiento, de la longevidad de la solución y sus componentes, de la distancia de los suministradores a la obra, etc. Es decir, que la evaluación medioambiental de soluciones constructivas requiere de una metodología con rigor científico.

Entre las metodologías aceptadas por la comunidad científica para evaluar el impacto medioambiental, el Análisis de Ciclo de Vida (ACV) resulta ser la más idónea por tratarse de un procedimiento analítico centrado en la evaluación del ciclo de vida completo de un proceso o producto, que trata los aspectos ambientales e impactos ambientales potenciales (por ejemplo, el uso de recursos y las consecuencias ambientales de las emisiones) a lo largo de todo el ciclo de vida de un producto desde la adquisición de la materia prima, pasando por la producción, uso, tratamiento final, reciclado, hasta su disposición final (12). Además el ACV está regulado por las normas UNE EN ISO 14040 y 14044 y es 
un sistema complejo que permite realizar estudios rigurosos y catalogar los resultados de forma objetiva, siempre que su aplicación sea llevada a cabo por personal cualificado.

Parece lógico considerar la necesidad de contar con programas informáticos o bases de datos informatizadas que faciliten la aplicación del ACV a materiales de construcción y a soluciones constructivas concretas, de manera que los proyectistas puedan tomar decisiones acertadas en lo referente al impacto ambiental de las soluciones constructivas desde la fase de diseño. Existe una gran variedad de herramientas para evaluar los componentes del edificio y los edificios completos que cubren todas las fases del ciclo de vida de los edificios y tienen en cuenta aspectos diferentes, además de ser aplicables a diferentes ámbitos, global, nacional, o local (13).

El objetivo del presente artículo es, de un lado, realizar un estado del arte de las herramientas existentes a nivel internacional de apoyo al diseñador para la selección de materiales y soluciones constructivas en la fase de diseño, de manera que se puedan identificar, evaluar y prevenir los impactos ambientales antes de que se produzcan, con la intención de dar un enfoque preventivo al proceso productivo, y de otro, realizar una evaluación de tales herramientas para concluir en una serie de directrices para una futura herramienta española. El presente artículo se centra más en los aspectos generales de funcionalidad de las herramientas estudiadas, que en cuestiones específicas de impactos considerados y formas de obtención de dichos impactos, porque entendemos, en la línea del enfoque global de ecodiseño marcado en la norma UNE-EN ISO ISO 14006:2011, que el diseño sostenible no sólo se trata de un problema de selección de materiales o sistemas constructivos, sino que constituye un proceso a través del cual el proyectista debe definir unos objetivos de desempeño ambiental del edificio previamente planificados y acordados con el promotor, que permitan la evaluación y seguimiento de los logros alcanzados en las diferentes fases del edificio que se proyecta.

\section{METODOLOGÍA DE INVESTIGACIÓN}

Ante la variedad de herramientas que proponen los diferentes organismos, países, centros de investigación etc., los estudios y trabajos de investigación publicados con información detallada de las herramientas y los numerosos artículos científicos (13) (14) (15) (16) (17) (18) (19) (20) (21) (22), se ha llevado a cabo una selección de herra- mientas y un posterior análisis en dos fases de la bibliografía y herramientas existentes, 1. Pasos seguidos en la investigación. según el esquema de la Figura 1.

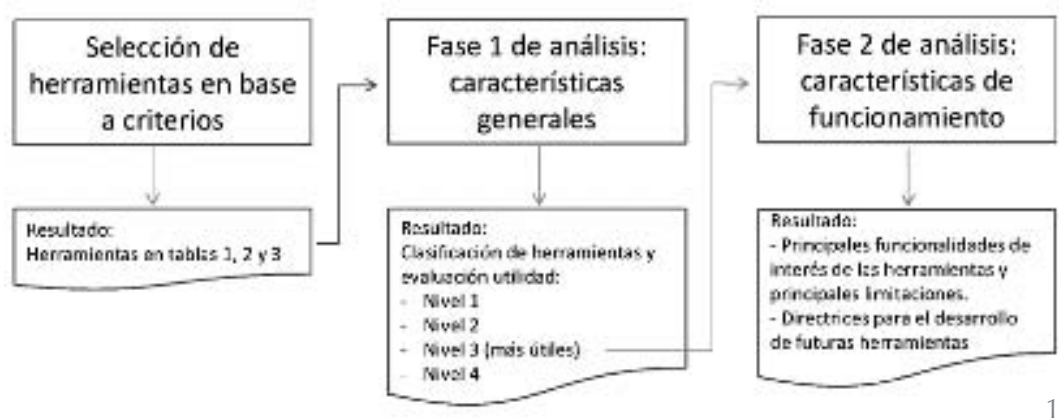

\subsection{Selección de herramientas}

En esta fase, se consideraron todas las herramientas de ACV que podían servir de apoyo al proyectista de las que había suficiente información accesible a través de publicaciones científicas y páginas web de diciembre de 2009 a junio de 2010.

Los criterios de selección de herramientas son los siguientes:

- Únicamente se han seleccionado herramientas de evaluación, basadas en el análisis de ciclo de vida (ACV).

- Que puedan servir de apoyo para diseñadores de construcción, en las que el objeto de evaluación sean materiales, soluciones constructivas o edificios completos.

- Se han tenido en cuenta las herramientas en las que los límites incluyan los procesos de la cuna a la tumba; las fases sugeridas por el Athena Institute (23) para los productos de la edificación son extracción de recursos, fabricación de productos, puesta en obra, ocupación/mantenimiento, demolición/deconstrucción, reciclado/ reutilización/retirada.

\subsection{Fase 1 de análisis: clasificación de las herramientas}

De cada herramienta se ha realizado una breve descripción y se han tenido en cuenta los siguientes criterios de análisis:

- Los métodos de evaluación de los impactos.

- Las bases de datos que utiliza.

- La forma de mostrar los resultados.

- Los idiomas en los que está disponible.

- Aspectos positivos de la herramienta para el apoyo al diseñador, tales como:

- Que cuente con datos específicos para edificación o construcción.

- Que sea exclusiva para edificación.

- Que esté desarrollada expresamente para la fase de diseño.

- Que utilice una base de datos modificable o ampliable.

- Que sea fácil de usar por un proyectista. 
Estos criterios de análisis han sido utilizados para clasificar, asimismo, las herramientas en cuatro niveles:

- Un primer nivel estará formado por herramientas genéricas de ACV, que se caracterizan por ser difíciles de usar por un proyectista por el tiempo y conocimientos requeridos, y por no ser exclusivas para edificación.

- Un segundo nivel estará formado por bases de datos de ACV de productos de construcción, desarrolladas expresamente para su uso en la fase inicial del diseño, pero que resultan difícil de utilizar por un proyectista.

- Un tercer nivel estará formado por herramientas de ACV exclusivas para la edificación, desarrolladas expresamente para su uso en la fase inicial del diseño.

- Un cuarto nivel formado por herramientas de certificación global de edificios, que no han sido desarrolladas para la fase inicial de diseño, sino que son de aplicación en fases avanzadas de diseño o cuando el proyecto ya está finalizado.

\subsection{Fase 2 de análisis: estudio de las herramientas específicas para el ACV en edificación}

Una vez identificados los tipos de herramientas, en esta segunda fase se estudian las consideradas más apropiadas para apoyo al proyectista, las de tercer nivel. No se intentan analizar los modelos de cálculo interno sino destacar los aspectos más relevantes en cuanto a las funcionalidades de mayor interés para el proyectista, teniendo en consideración los siguientes aspectos:

- Alcance de la herramienta.

- Impactos ambientales considerados.

- Bases de datos.

- Funcionamiento de la herramienta.

- Obtención de resultados.

- Valoración de la herramienta como apoyo al proyectista.

- Limitaciones en la aplicabilidad de la herramienta.

\section{RESULTADOS}

\subsection{Selección de herramientas}

Las Tablas* 1, 2 y 3 recogen las herramientas seleccionadas, que han sido estudiadas

\subsection{Fase 1 de análisis: clasificación de las herramientas seleccionadas}

Las herramientas del primer nivel, herramientas genéricas de ACV, consideradas son: AistLCA, eVerDEE, Gabi 4, JEMAI-L-LCA, Lcapix,
Las herramientas de segundo nivel (bases de datos de ACV de productos de construcción) son:

- Inventory of Carbon and Energy (ICE) (24), de la Universidad de Bath, en Reino Unido (http://www.bath.ac.uk/mech-eng/ sert/embodied/). Es una hoja de cálculo, en inglés, que incluye más de 400 valores de energía embebida/carbono embebido de aproximadamente 170 tipos de materiales diferentes de edificación.

- Fiches de Déclaration Environnementale et Sanitaire (FDES) des Produits de construction, de Francia (http://www.inies.fr/). Es una base de datos online, en francés, que incluye más de 5000 fichas de declaración ambiental y características sanitarias de productos de construcción en el mercado francés.

- Ecoetiquetado de productos de la construcción (DAPc), de diversos países. Es una ecoetiqueta tipo III denominada declaración ambiental de producto (DAP o Environmental Product Declaration, EPD) sobre los productos de la construcción por lo que sus siglas son DAPc. Dichas etiquetas están basadas en estudios de ACV verificados de forma independiente y realizados bajo unas directrices consensuadas, permitiendo una comparación equitativa entre distintos productos porque sigue unas reglas específicas para cada categoría de producto, denominadas Reglas de Categoría de Producto $(\mathrm{RCP})$. En España, el proyecto del sistema DAPc se inició en febrero del año 2008. Por el momento, se han realizado las pruebas pilotos de 6 productos de la construcción que se pueden consultar en http://es.csostenible.net/productes/ productos/

La Tabla 2 recoge las herramientas estudiadas de tercer nivel, herramientas específicas para el ACV en edificación, en las que se incluyen: ATHENA® Estimator, BEAT 2002, BeCost, BEES, EcoBat, EcoCalculator, EcoEffect, Eco-Quantum, EcoSoft WBF, Envest2, EQUER, LCAid, LEGEP, LISA, Metabase y TCQ2000, OFEN.

Las herramientas de certificación sostenible de edificios, de cuarto nivel, consideradas son: Breeam, Casbee, Enlace, GBTool, Green Globes ${ }^{\mathrm{TMB}}$, Leed, Verde (Tabla 3).

La clasificación propuesta nos permite identificar la validez de las herramientas en lo referente al apoyo al diseñador en la selección de sistemas constructivos. Aunque todas las herramientas descritas podrían ser utilizadas para la evaluación medioambiental de sistemas constructivos, y en este sentido pueden considerarse herramientas 
de apoyo al diseñador para la toma de decisiones, las herramientas más útiles para el proyectista en las fases iniciales de diseño son las que permiten clasificar las herramientas como de tercer nivel (herramientas específicas para el ACV en edificación), es decir, aquellas que son exclusivas para edificación, son fáciles de usar por un proyectista por facilitar el uso de las mismas a nivel de sistema constructivo o edificio y se han desarrollado expresamente para la fase de diseño.

Las herramientas catalogadas dentro del primer nivel no son muy operativas para ser utilizadas en la fase de diseño por un proyectista debido a su complejidad. El proyectista tendría que abordar un amplísimo rango de cuestiones, tomando conciencia de muchas consideraciones no solo en los aspectos relativos a la sostenibilidad (25). Además, cabe decir que estas herramientas contienen pocos datos de materiales de construcción. Es verdad que se podrían generar estos datos con su uso, y a su vez, generar información de los sistemas constructivos a partir de los materiales, su puesta en obra, mantenimiento, etc. Sin embargo, esta forma de trabajar es impensable para los proyectistas, que no tienen ni los conocimientos ni el tiempo para abordar un proyecto de diseño de un edificio aplicando el Análisis de Ciclo de Vida desde los materiales o las materias primas.

Las herramientas catalogadas dentro del segundo nivel son útiles a nivel de producto de la construcción, y por tanto, para las empresas fabricantes, pero su uso a nive de sistema constructivo es más complicado porque requiere la recopilación adicional de información relativa a las fases de ejecución, uso y mantenimiento de la solución constructiva en la que se aplican los productos con declaración ambiental.

Las herramientas catalogadas dentro del cuarto nivel son programas/esquemas de certificación de edificios que ya están siendo ampliamente utilizados en los mercados más exigentes. Estos introducen una aproximación al ACV para la evaluación y certificación de edificios completos, que sirven para fomentar el diseño sostenible a largo plazo y permiten el establecimiento de parámetros que mejoran la calidad (17). Sin embargo, no son útiles en las fases iniciales del diseño, porque para poder utilizarlos el edificio ha de estar definido prácticamente en su totalidad. Estas herramientas se podrían beneficiar de las herramientas de segundo nivel, de manera que alimenten sus bases de datos con fuentes más exactas.

\subsection{Fase 2 de análisis: estudio de las herramientas específicas para el ACV en edificación}

Las herramientas seleccionadas son Athena, Impact Estimator, Beat 2002, BeCost, BEES, Ecobat, Ecocalculator, EcoEffect, EcoQuantum, EcoSoft, Envest2, EQUER, LCAid, LEGEP, LISA y OFEN. La Tabla 4 muestra los resultados del análisis, que a continuación comentamos, organizados en:

- Funcionalidades de mayor interés de las herramientas.

- Valoración de las herramientas como apoyo al proyectista

- Limitaciones de las herramientas.

\subsubsection{Funcionalidades de mayor interés de las herramientas}

Las funcionalidades de mayor interés observadas en estas herramientas son:

- La integración de los requisitos técnicosfuncionales de sostenibilidad en la edificación para ayudar a los proyectistas en la selección de materiales y de soluciones constructivas, teniendo en cuenta los requisitos integrados.

- La posibilidad de evaluar en la fase inicial del diseño, los impactos medioambientales mayoritariamente aceptados, para saber qué materiales o qué soluciones constructivas son responsables de un mal comportamiento medioambiental y poder sustituir un material por otro o de seleccionar una solución óptima sin tener que modificar el diseño una vez realizado.

- La integración de la información obtenida en las bases de datos de la construcción y catálogos de elementos constructivos incluyendo criterios económico-ambientales.

- La obtención de puntuaciones que se puedan cuantificar sobre una base de unidad funcional, mediante indicadores, que permitan comparar resultados o acreditar el cumplimiento de requisitos medioambientales de cara a la obtención de licencias, subvenciones etc.

\subsubsection{Valoración de las herramientas como apoyo al proyectista}

En el estudio de cómo pueden ayudar estas herramientas al proyectista en la toma de decisiones, hemos llegado a las siguientes conclusiones:

- Estas herramientas incorporan bases de datos de materiales y/o soluciones constructivas que incluyen datos de impactos ambientales, lo que permite al proyectista conocer los resultados reales del impacto 
ambiental de las soluciones constructivas adoptadas en fase de diseño para poder adoptar soluciones preventivas.

- Facilitan la labor del proyectista mediante la caracterización, comparación y selección de alternativas no solo a nivel de materiales, sino también a un nivel intermedio entre el material y el edificio, combinando diferentes materiales que formen parte de una solución constructiva. Se ha de tener en cuenta que un sistema constructivo puede tener un componente de alto impacto, pero si su contribución es en una proporción pequeña puede estar compensado por otros componentes relativamente benignos.

- Algunas de estas herramientas permiten la exportación de datos de manera que el proyectista puede definir soluciones constructivas y luego utilizarlos en otro proyecto.

- Pueden servir para verificar el cumplimiento de los objetivos medioambientales que se planteen.

- Permiten comprobar los efectos del cuidado, mantenimiento y reparación de las acciones sobre el medio ambiente.

- Algunas de estas herramientas permiten realizar una valoración económica de los costos medioambientales.

\subsubsection{Limitaciones de las herramientas}

Todas estas herramientas presentan limitaciones:

- Difícilmente son aplicables a la construcción española.

- Sólo unas pocas consideran los consumos de agua (tan importantes en un país como el nuestro con estrés hídrico).

- Pocas contienen información por defecto de la fase de ejecución.

- Algunas no contienen información por defecto de los impactos de la fase uso y mantenimiento, lo cual es considerado un inconveniente por la comunidad científica (26) (27).

\section{CONCLUSIONES}

El ACV es tal y como se ha descrito en la introducción de este artículo, una metodología rigurosa, que está perfectamente definida. El proyectista dispone mediante el análisis de ciclo de vida de una herramienta que le permite evaluar los impactos ambientales en las primeras etapas del diseño.

Ahora bien, se ha definido el ACV como un proceso exhaustivo, muy laborioso y de gran complejidad, ya que las consideraciones necesarias para su aplicación son muy numerosas. El ACV puede aplicarse a un solo material (el cemento, la madera...), a un producto (una carpintería de aluminio), a una solución constructiva, (una cubierta plana), o a un edificio completo (7), pero su aplicación requiere mucho tiempo y una cualificación que normalmente resulta incompatible con las condiciones de trabajo del proyectista. Precisamente, esta circunstancia es la que ha motivado la búsqueda y análisis de aquellas herramientas que, como se ha visto, pueden resultar más o menos aptas para apoyar al proyectista en la toma de decisiones, en la fase inicial del diseño manteniendo el rigor del ACV.

De entre las herramientas encontradas, que hemos clasificado en herramientas genéricas de ACV, bases de datos de productos de la construcción, herramientas específicas para el ACV en edificación, y herramientas para la certificación de la sostenibilidad de edificios, son las específicas para el ACV en edificación las que más pueden servir de ayuda a los proyectistas.

Sin embargo, todas las herramientas analizadas presentan limitaciones. Un importante hándicap es que ninguna de estas herramientas es de aplicación en otro país que en el generado, ya que el nivel de impacto ambiental causado por los materiales y soluciones constructivas puede variar en función del territorio, debido a la ubicación geográfica de la obtención de las materias primas y de los centros de transformación con relación a la situación del edificio que se ha de construir. Sólo una de estas herramientas es española, estando sus datos ambientales basados en el análisis de bases de datos europeas, por lo que su aplicabilidad al caso español es reducida.

Otro inconveniente de estas herramientas es que pocas consideran los consumos de agua (tan importante en un país como el nuestro con estrés hídrico).

Para disponer en España de una herramienta rigurosa que permita catalogar o baremar las diferentes soluciones constructivas a partir de los análisis de ciclo de vida sería necesario, en primer lugar, Ilegar a un acuerdo sobre una metodología con rigor científico que permita unívocamente aplicar la metodología de análisis de ciclo de vida a los sistemas constructivos (como ya se ha hecho para los productos de construcción a través del sistema de DAPc), utilizando indicadores de sostenibilidad reconocidos, para ponderar los resultados y poder generar bases de datos de materiales y de soluciones constructivas con una valoración rigurosa.

Como conclusión final, se derivan de los análisis realizados las directrices para el desarrollo de una herramienta de asistencia 
en la selección de soluciones constructivas en España para una edificación sostenible, que se enuncian a continuación:

- Que sirva para la evaluación en la fase inicial del diseño de materiales de construcción, sistemas constructivos y edificios.

- Que esté basada en el ACV completo, desde la extracción de recursos, fabricación, transporte, construcción, mantenimiento, demolición hasta la gestión de residuos.

- Que considere los consumos energéticos de la fase uso achacables a la envolvente.

- Que tenga en cuenta los consumos de agua.

- Que pueda calcular los costes ambientales durante toda la vida útil del edificio.

- Que utilice algún método de evaluación de impactos científicamente reconocido.

- Que cuente con bases de datos de energía, de combustibles, de materiales, de productos de construcción, de soluciones constructivas y de edificios completos, ampliables y compatibles con el formato SPOLD.

- Que tenga en cuenta la ubicación geográfica, la zona climática, la orientación, el transporte etc.

- Que permita seleccionar datos relativos a la tipología, geometría y uso del edificio.

- Que permita especificar los impactos a evaluar y obtener los resultados de la evaluación de cada impacto por separado.
- Que valore los impactos utilizando indicadores medioambientales.

- Que ofrezca resultados en forma numérica o en forma gráfica.

- Que cuente con un sistema de valoración de resultados mediante puntuaciones que se puedan cuantificar sobre la base de unidad funcional.

- Que sea intuitiva y fácil de usar por un proyectista.

- Que muestre los impactos ambientales pormenorizadamente en función de diferentes opciones de diseño, ya constituyan estos materiales, productos, soluciones constructivas o edificios completos, para conocer la contribución de cada objeto de la evaluación a los impactos ambientales del edificio

- Que realice la evaluación de los impactos en tiempo real, para comparar entre diferentes opciones en fases tempranas del diseño y poder elegir entre una u otra.

- Que pueda importar datos de CAD.

- Que sirva para verificar el cumplimiento de exigencias medioambientales para la obtención de licencias, permisos, subvenciones etc.

- Que permita calcular el coste económico de la edificación en base a las soluciones ambientales seleccionadas.

- Que lleve asociada una base de datos que permita realizar conjuntamente las mediciones y presupuestos del proyecto.

\section{REFERENCIAS}

(1) Brundtland, G. (1987). Our common future. Report of the World Commission on Environment and Development. Transmitted to the General Assembly as an Annex to document A/42/427-Development and International Co-operation: Environment.

(2) Oteiza, I., Alonso, C. (2008, 24 de mayo). Análisis y revisión de herramientas para evaluación de la sostenibilidad de la construcción. Actas de las II Jornadas de Investigación en Construcción, pp. 1149-1166. Madrid.

(3) Real Decreto 314/2006, de 17 de marzo, por el que se aprueba el Código Técnico de la Edificación.

(4) Real Decreto 47/2007, de 19 de enero, por el que se aprueba el Procedimiento básico para la certificación de eficiencia energética de edificios de nueva construcción.

(5) Real Decreto 105/2008, de 1 de febrero, por el que se regula la producción y gestión de los residuos de construcción y demolición.

(6) Estrategia temática sobre el uso sostenible de los recursos naturales. Comunicación de la Comisión de 21 de diciembre de 2005 - COM (2005) 670.

(7) González, M.J. (2004, 27 de febrero). El papel de los materiales de Construcción en la arquitectura sostenible. Actas de las III Jornadas abulenses de energías renovables y medio ambiente. Taller de arquitectura de bajo impacto ambiental. Ávila.

(8) Castro-Lacouture, D., Sefair, J., Flórez, L., Medaglia, A. (2009). Optimization model for the selection of materials using a LEED-based green building rating system in Colombia. Building and Environment, 44(6): 1162-1170. doi: 10.1016/j.buildenv.2008.08.009.

(9) Ferrer, I. (2007). Contribución metodológica en técnicas de diseñar para fabricación. (Tesis doctoral). Universidad de Girona.

(10) López-Mesa, B., Gallego, T., Mulet, E., Pitarch, A., Tomás, A. (2007, 28-31 de agosto). Exploring the need for an evaluation model to assist in the eco-efficient selection of building systems. Proceedings of the 16th International Conference on Engineering Design 2007 (ICED07). París.

(11) "100 Materiales Sostenibles", ICARO del Colegio Territorial de Arquitectura de Valencia, 2003. 
(12) Cebrián-Tarrasón, D., Garraín, D., París, A., Vidal, R. (2009, 8-10 de julio). ACV libre: la Utilización del ELCD en la Fase de Diseño. Actas del XIII Congreso de Ingeniería de Proyectos (AEIPRO). Badajoz.

(13) Haapio, A., Viitaniemi, P. (2008). A critical review of building environmental assessment tools. Environmental Impact Assessment Review, 28(7): 469-482. doi: 10.1016/j. eiar.2008.01.002.

(14) ANNEX 31, International Energy Agency. Retrieved June 16, 2010 from the World Wide Web: http://www.uni-weimar.de/scc/PRO/survey.html.

(15) Eldridge, C. (2002). Lcaid ${ }^{\mathrm{T}}$ Software: Measuring Environmental Performance Of Buildings. NSW Department of Public Works and Services, Sydney, Australia.

(16) Forsberg, A., Malmborg, F. (2004). Tools for environmental assessment of the built environment. Building and Environment, 39(2): 223-228. doi: 10.1016/j.buildenv.2003.09.004.

(17) Fowler, K., Rauch, E. (2006). Sustainable Building Rating Systems Summary. Completed by the Pacific Northwest National Laboratory for the U.S. Department of Energy, U.S.

(18) Kohler, N., Wagnwe, A., Luetzkendorf, T., König, H. (2005, 27-29 de septiembre). Life Cycle assessment of passive buildings with LEGEP - a LCA-tool from Germany. World Sustainable Building Conference, Tokyo.

(19) Lippiatt, B., Boyles, A. (2001). Using Bees to select Cost-Effective Green Products. International Journal of LCA, 6(2): 76-80.

(20) Macías, M., García Navarro, J. (2010). Metodología y herramienta VERDE para la evaluación de la sostenibilidad en edificios. Informes de la Construcción, 62(517): 87-100, doi: 10.3989/ic.08.056.

(21) Seo, S., Tucker, S., Ambrose, M., Mitchell, P., Wang, C-H. Technical Evaluation of Environmental Assessment Rating Tools. Forest and Wood Products Research and Development Corporation. Australia.

(22) Yuan, W. (2005). Comparison of two sustainable building assessment tools applied to Holmen project in Stockholm. (Masther thesis). Royal Institute of Technology, Stockholm, Sweden.

(23) Athena Institute. LCA model. Retrieved June 16, 2010 from http://www.athenasmi.ca

(24) Hammond, G. P., Craig I. J. Inventory of Carbon and Energy (ICE), Sustainable Energy Research Team (SERT), University of Bath, 2006. Retrieved June 10, 2010 from http:// www.bath.ac.uk/mech-eng/sert/embodied/

(25) Howarth, G., Hadfield, M. (2006). A sustainable product design model. Materials and Design, 27(10): 1128-1133. doi: 10.1016/j.matdes.2005.03.016.

(26) Malin, N. (2005). Life cycle assessment for whole buildings: seeking the holy grail. Building Design and Construction, pp. 6-11.

(27) Ruá, M.J., Vives, L., Civera, V., López-Mesa, B. (2010, 15-16 de febrero). Aproximación al cálculo de la eficiencia energética de fachadas ventiladas y su impacto ambiental. Actas del XI Congreso mundial de la calidad del azulejo y del pavimento cerámico QUALICER. Castellón. 


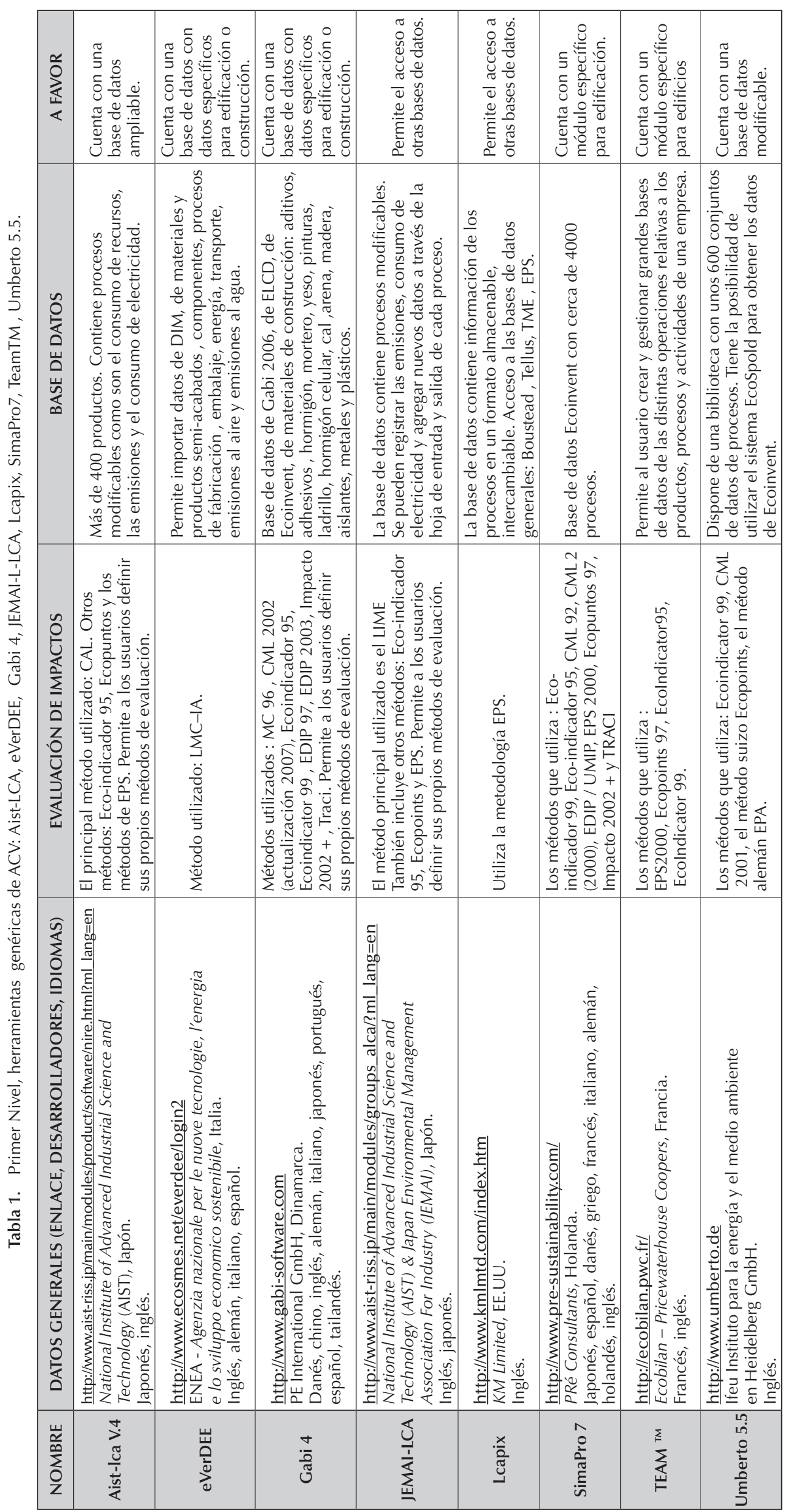




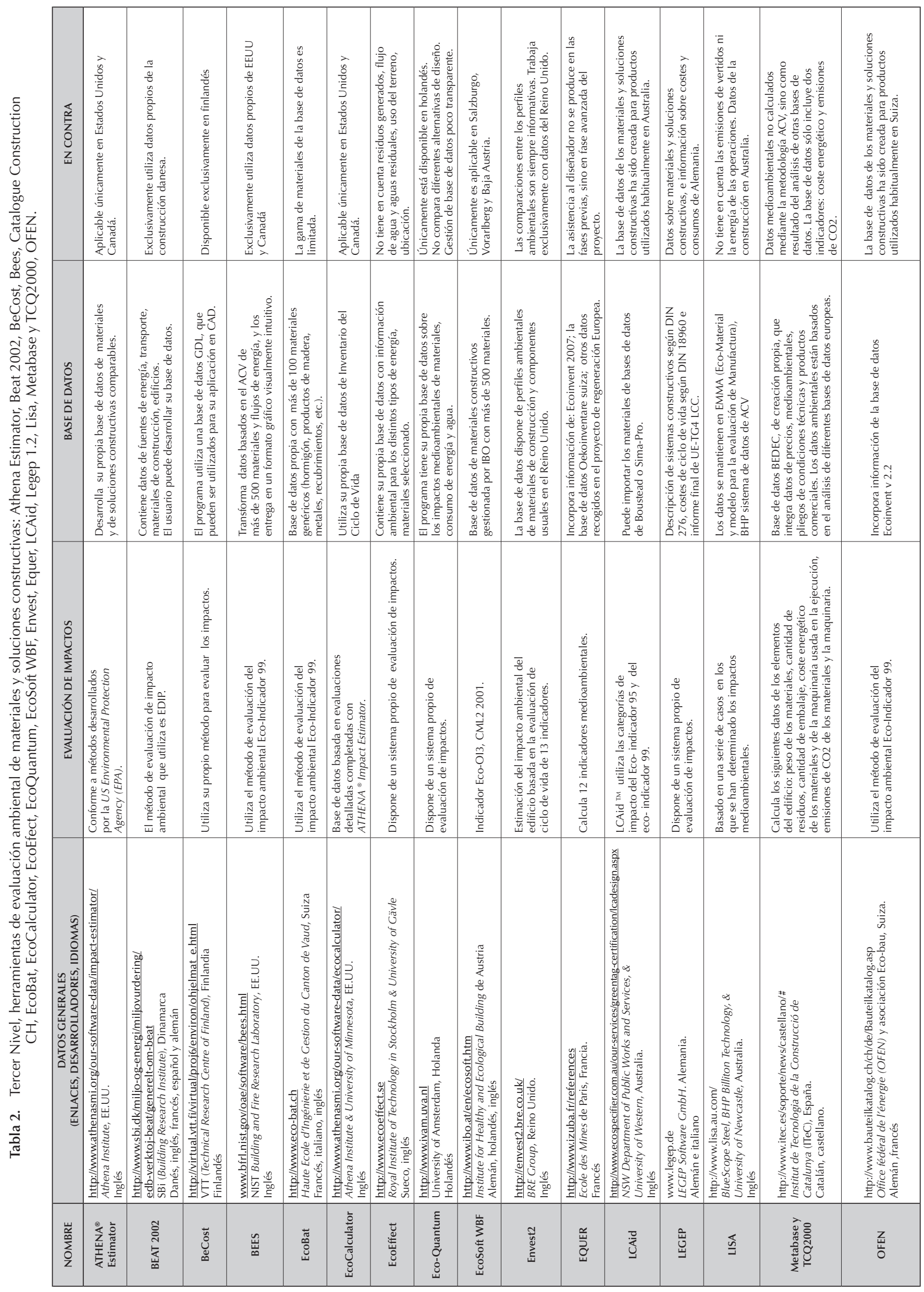




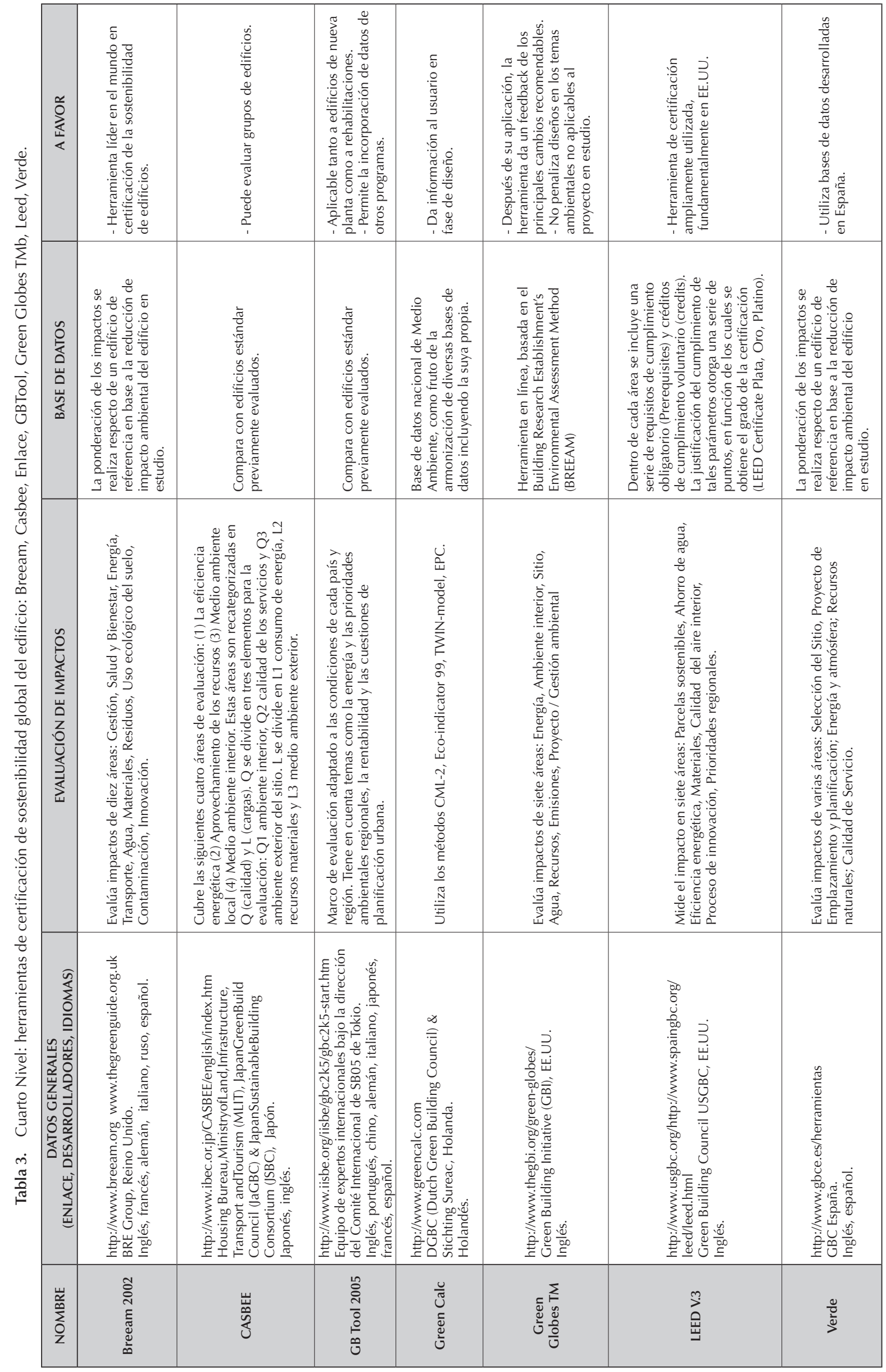




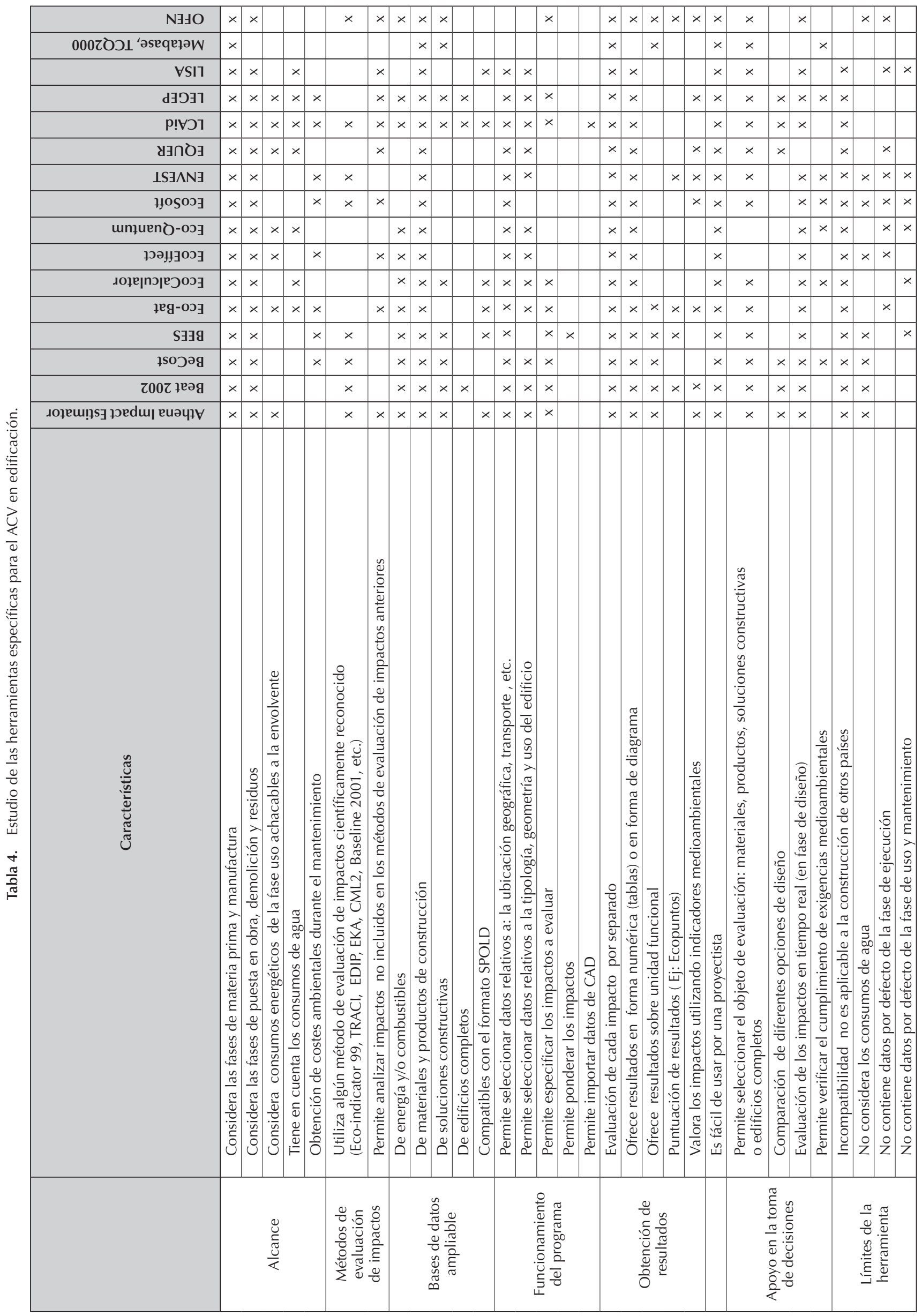

